\section{Does global warming make Triton blush?}

Neptune's largest moon, Triton, is one of two satellites in the Solar System that are currently geologically active. At least two geyser-like plumes were observed by the Voyager 2 spacecraft in 1989, and dozens of streaky deposits hint at the existence of many more ${ }^{1}$. Triton also exhibits complex seasonal changes in its 165-year journey about the Sun ${ }^{2}$. Because Triton's atmosphere transports volatiles (primarily nitrogen and methane) during this seasonal cycle, its atmospheric pressure may fluctuate by up to an order of magnitude over decades ${ }^{3}$. Photometric measurements of its albedo and colour over half a century show that seasonal volatile transport has occurred ${ }^{4}$. There have also been indications that more extreme, short-lived changes, perhaps due to geological events, have occurred on Triton. An anomalously red spectrum was reported for Triton in 1977 (refs 5,6), and global warming has now been observed ${ }^{7}$.

We obtained four spectra of Triton between 0.35 and $0.95 \mu \mathrm{m}$ using the 200inch Hale telescope and the double spectrograph at Palomar Mountain Observatory on 23 October 1997. The spectral resolution was $10 \AA$ between 0.35 and $0.55 \mu \mathrm{m}$, and $5 \AA$ between 0.55 and $0.95 \mu \mathrm{m}$. We analysed the data according to standard procedures, and the 4-arcsecond slit was aligned to minimize the possibility of differential refraction. Our reduced spectrum for Triton is

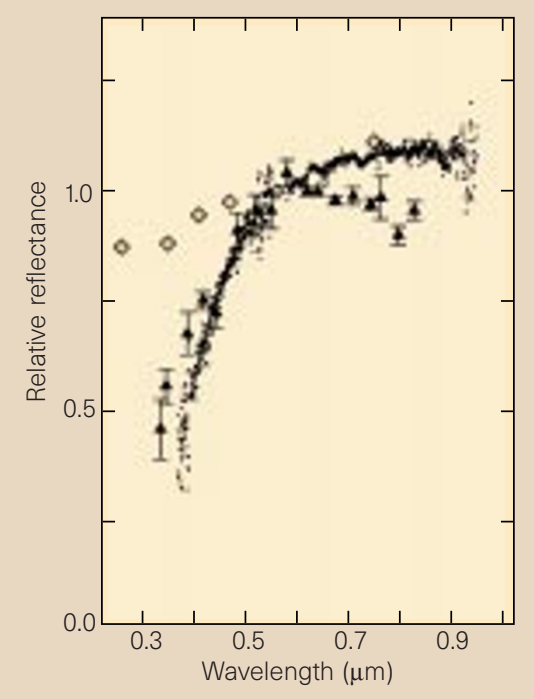

Figure 1 Spectral reflectivity of Triton. Our data were obtained in October 1997 at the Palomar Mountain Observatory (dots). The spectrum from the Voyager Imaging Science Subsystem and Photopolarimeter Subsystem (diamonds) is representative of Triton's colour in 1990 and just before, whereas the 1977 data $^{5,6}$ (triangles) represent a previous observation of a reddening of Triton's spectrum. compared with previous observations in Fig. 1: it is significantly different from that normally observed, but is similar to the anomalous 1977 spectrum ${ }^{5,6}$. The upper limit for the timescale for our observed spectrum is seven years (the period between the last published spectrum in the range 0.35-0.55 $\mu \mathrm{m}$ (ref. 4) and October 1997).

A global temperature increase of nearly $2 \mathrm{~K}$ was observed on Triton between 1989 and November 1997 (ref. 7). Our observations indicate that this change may be due to deposition of material from a geological process on Triton. Both the spectral changes and the global warming may have been caused by a triggering event, such as massive venting, causing the deposition of dark, red material onto the surface of Triton. The spectrum of the streaks that appear to be plume deposits are indeed darker and redder than Triton as a whole $^{8}$. The most volatile, fresh, blue nitrogen ice may initially sublimate to expose a lower layer of red photolysed methane ice ${ }^{9}$, perhaps causing an additional temperature rise. Of the mapped geological regions, our spectrum is most like that of a region that is probably rich in irradiated methane clathrate ${ }^{8}$. Our spectrum has a more pronounced methane absorption band at about $0.73 \mu \mathrm{m}$ than a spectrum obtained in 1989 (ref.10).

Whatever the triggering mechanism(s), Triton's surface will warm significantly as its visual and near-ultraviolet albedo decrease. Triton's Bond albedo ( $A=p q$, where $p$ is the geometric albedo and $q$ is the phase integral) is among the highest of any body in the Solar System. Its energy balance is therefore particularly sensitive to small variations in its spectral albedo. At the time of the Voyager 2 encounter, $A$ was 0.89 in the visual region of the spectrum, where most of the solar flux occurs ${ }^{1}$. Because $T \approx(1-A)^{1 / 4}$, where $T$ is the temperature, a decrease of only about $3 \%$ in the Bond albedo would account for the observed temperature increase ${ }^{7}$. Increases in the absolute (geometric) albedo, which our observations were not designed to measure, may mitigate some of the decrease in the Bond albedo.

Models for global seasonal change on Triton that include only the effects of solar insolation could underestimate global temperature changes and the atmospheric loading of volatiles.

Bonnie J. Buratti, Michael D. Hicks,

Ray L. Newburn Jr

Jet Propulsion Laboratory,

California Institute of Technology,

Palomar Observatory,

4800 Oak Grove Drive 183-501,

Pasadena, California 91109, USA

e-mail:bburatti@ocelot.jpl.nasa.gov

1. Smith, B. et al. Science 246, 1422-1449 (1989)

2. Trafton, L. Icarus 58, 312-324 (1984).

3. Brown, R. H., Johnson, T. V., Goguen, J. D., Schubert, G. \& Ross, M. N. Science 251, 1465-1467 (1991).
4. Buratti, B., Goguen, J. D., Gibson, J. \& Mosher, J. Icarus 110, 303-314 (1994).

5. Cruikshank, D. P., Stockton, D., Dyck, H. M., Becklin, E. E. \& Macy, W. Jr Icarus 40, 104-114 (1979).

6. Bell, J., Clark, R. N., McCord, T. B. \& Cruikshank, D. P. Bull. Am. Astron. Soc. 11, 570 (1979).

7. Elliot, J. et al. Nature 393, 765-767 (1998).

8. McEwen, A. J. Geophys. Res. 17, 1765-1768 (1990).

9. Thompson, W. R., Murray, B., Khare, B. N. \& Sagan, C. J. Geophys. Res. 92, 14933-14947 (1987).

10. Grundy, W. \& Fink, U. Icarus 93, 379-385 (1991).

\section{Shikimate pathway in apicomplexan parasites}

The discovery of plastids in apicomplexan parasites $^{1-3}$ raised the possibility that these organelles might harbour plastid-specific metabolic activities that could be blocked by therapeutic agents. Ideally, these agents would inhibit the parasites without harming their vertebrate hosts. Roberts et al. ${ }^{4}$ have made the promising dicovery that Apicomplexa are sensitive to the herbicide glyphosate (better known by its trade names of RoundUp, Zero or Tumbleweed), which is an inhibitor of the enzyme 5enopyruvyl shikimate 3-phosphate synthase. They suggested that production of aromatic amino acids by the pathway involving this enzyme, the shikimate pathway, might be an essential function of the apicomplexan plastid, but here we present evidence that this pathway actually operates in the cytosol of Apicomplexa.

The shikimate pathway occurs in prokaryotes, fungi and the plastids of plants and algae, but not in vertebrates ${ }^{5}$. In plants, the shikimate-pathway enzymes, like other nuclear-encoded plastid proteins, are posttranslationally targeted to the plastid by means of an amino-terminal leader sequence. Apicomplexan proteins destined for the plastid also include substantial leader peptides, which are sufficient for the transport of reporter proteins across all four membranes of the plastid of the apicomplexan parasite Toxoplasma ${ }^{6}$.

In contrast to the plant enzymes, there is no evidence that apicomplexan shikimate enzymes are localized in the plastid, and neither do the Toxoplasma and Plasmodium chorismate synthase genes encode leader peptides (see Fig. 2 of ref. 4). This absence of a leader strongly suggests that the apicomplexan chorismate synthase proteins function in the cytosol and not the plastid. This would not be unprecedented, for the shikimate pathway operates in the cytosol of fungi ${ }^{7}$. Moreover, the fungal shikimate pathway is sensitive to glyphosate . $^{7}$

Phylogenetic analysis of chorismate synthase (Fig. 1) is consistent with this idea, as the apicomplexan proteins are most closely related to fungal cytosolic proteins, whereas plastid-targeted proteins from plants group 


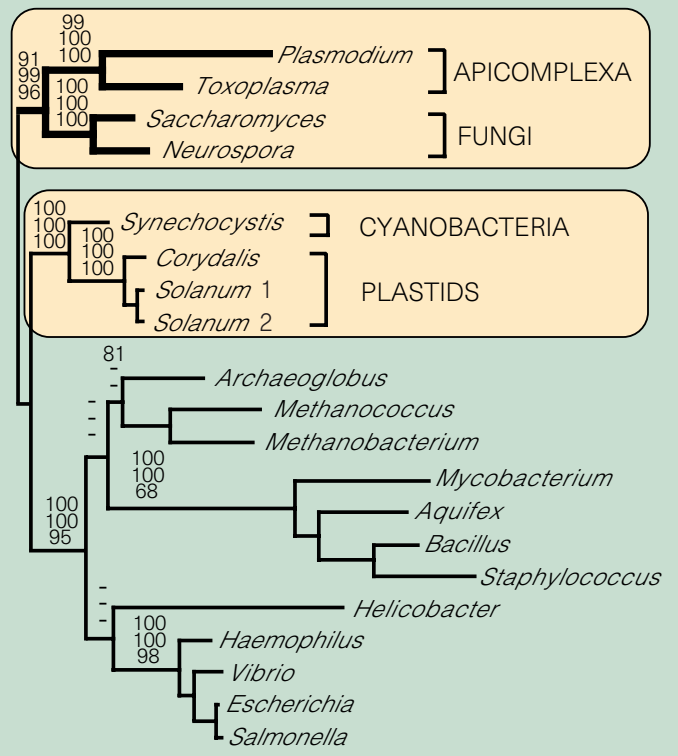

Figure 1 Phylogeny of chorismate synthase. The figure shows a Fitch-Margoliash tree, based on maximum-likelihood distances corrected by the Jones, Taylor and Thornton substitution matrix. Other methods (neighbour-joining, maximum parsimony, protein maximum likelihood, and quartet puzzling) all also support the grouping of fungi and Apicomplexa, and of cyanobacteria and plastids. Numbers at the nodes correspond to support for the major groups from (top to bottom): Fitch-Margoliash bootstrap, protein maximum-likelihood resampling estimated log-likelihood bootstrap, and per cent occurrence in quartet-puzzling steps. Dashes indicate support of less than 50 per cent.

with cyanobacteria, as expected. The grouping of the fungal/apicomplexan lineage with the cyanobacterial/plastid lineage, although curious, should not be mistaken for plastid origin because this tree is unrooted, the cytosolic genes do not branch specifically with plastids, and fungi lack plastids and indeed probably never had them.

The evolutionary origin of cytosolic shikimate-pathway enzymes in eukaryotes is unclear, as the distribution of the cytosolic pathway in eukaryotes is poorly understood and the sampling of sequences is limited to fungi and Apicomplexa. Nevertheless, in view of the phylogeny of chorismate synthase and the absence of leader peptides, there is no reason to believe that the shikimate pathway of Apicomplexa is either derived from or functions in the plastid.

Even if the shikimate pathway does take place in the cytosol in Apicomplexa, this does not detract from the importance of the sensitivity of these parasites to glyphosate. Vertebrate hosts of Apicomplexa lack this pathway, so it remains a specific chemo-therapeutic target. However, before glyphosate sensitivity can be exploited, we need to define the role, if any, of the plastid in the apicomplexan shikimate pathway.

Patrick J. Keeling* ${ }^{\star}$ Jeffrey D. Palmer ${ }^{\star}$, Robert G. K. Donald $\dagger$, David S. Roos $\dagger$, Ross F. Waller $\ddagger$, Geoffrey I. McFadden $\ddagger$

${ }^{*}$ Department of Biology,

Indiana University,

Bloomington, Indiana 47405, USA

$\dagger$ Department of Biology,

University of Pennsylvania,

Philadelphia, Pennsylvania 19104, USA

$\ddagger$ Plant Cell Biology Research Centre,

Botany School, University of Melbourne,

Parkville, Victoria 3054, Australia

1. Wilson, R. J. et al. J. Mol. Biol. 261, 155-172 (1996).

2. McFadden, G. I., Reith, M. E., Munholland, J. \& Lang-Unnasch, N. Nature 381, 482 (1996).
3. Köhler, S. et al. Science 275, 1485-1489 (1997).
4. Roberts, F. et al. Nature 393, 801-805 (1998).
5. Kishore, G. M. \& Shah, D. M. Annu. Rev. Biochem. 57, 627-663
(1988).
6. Waller, R. F. et al. Proc. Natl Acad. Sci. USA 95, 12352-12357
(1998).
7. Boocock, M. R. \& Coggins, J. R. FEBS Lett. 154, 127-133 (1983).

Roberts et al. reply - Although our study ${ }^{1}$ was stimulated by the discovery of the apicomplexan plastid, we did not claim to have provided proof that the shikimate pathway occurred in the plastid in apicomplexans. We believe that the site of this pathway remains an open question, despite the similar data emerging from our own ${ }^{2}$ and Keeling et al.'s phylogenetic analyses of our chorismate synthase gene sequences.

The premise for the phylogenetic analyses was that if the shikimate pathway occurs in the plastid, then these gene sequences are likely to show evolutionary relatedness to those in green algae, like the genes present on apicomplexan plastid DNA. However, phylogenetic analysis of chorismate synthases in the apicomplexans does not support this hypothesis; rather, it points to a closer similarity with fungal enzymes. From this, Keeling et al. conclude that the shikimate pathway is cytosolic (as it is in fungi).

This situation might have been expected if apicomplexans had evolved directly from a common fungal lineage, but this is not generally believed to be the case. At present, the best evidence available aligns apicomplexans more closely with ciliates and plants, with fungi as distant relatives.

Caution is needed in drawing conclusions about the subcellular locations of proteins from phylogenetic analysis. Such studies are fraught with difficulty because of the frequency with which horizontal gene transfer occurs during evolution, especially when the data are from a relatively small number of organisms. We believe that phylogenetic analysis is an inadequate tool for determining the location(s) of the shikimate pathway in apicomplexans: direct experimental evidence of the location of key proteins is required.

The two apicomplexan chorismate synthases do not have an obvious aminoterminal leader sequence like that found on plastid chorismate synthases of higher plants. However, apicomplexan protein sequences differ from all other reported chorismate synthases in that they have a number of large insertions. The function(s) of these insertions remain(s) unknown, but one of them might act as a targeting sequence, as proteins can be targeted to plastids even without an amino-terminal leader sequence ${ }^{3}$.

The location of the shikimate pathway in apicomplexan parasites remains an open question. A rigorous, practical approach should reveal that all, some or even none of the shikimate enzymes function within the plastid, or the pathway may turn out to operate both outside and inside the plastid in apicomplexans, as in Euglena ${ }^{4}$. Irrespective of where the pathway is located, however, it presents a prime target for antiparasitic agents. A cytosolic location would be more accessible to a target enzyme as fewer membranes would need to be crossed.

Craig W. Roberts $\dagger^{\star} \neq$, John Finnerty§, Jennifer J. Johnson ${ }^{\star}$, Fiona Roberts ${ }^{\star} \dagger$, Dennis E. Kyle\|, Tino Krellg,

John R Cogginsf, Graham H. Coombsg, Wilbur K. Milhous\|, Saul Tzipori\#,

David J. P. Ferguson $\_$,

Debopam Chakrabartif, Rima McLeod ${ }^{\star}$

${ }^{*}$ Michael Reese Hospital and Medical Center,

Chicago, Illinois 60616, USA

and the University of Chicago,

Chicago, Illinois 60637, USA

$\ddagger$ Department of Immunology,

University of Strathclyde, Glasgow G4 ONR, UK

$\S$ Department of Organismal Biology and Anatomy,

University of Chicago,

Chicago, Illinois 60637, USA

||Walter Reed Army Institute of Research,

Washington DC 20307-5100, USA

Institute of Biomedical and Life Sciences, University of Glasgow, Glasgow G12 8QQ, UK

\#Tufts University School of Veterinary Medicine,

Boston, Massachusetts 01536, USA

is John Radcliffe Hospital, Oxford University, Oxford OX3 9DU, UK

SUniversity of Central Florida,

Orlando, Florida 32816-2360, USA

$\dagger$ Present addresses: University of Strathclyde,

Glasgow, UK (C.W.R.); University of Glasgow, Glasgow, UK (F.R.)

\footnotetext{
1. Roberts, F. et al. Nature 393, 801-805 (1998).

2. Roberts, C. W. et al. Keystone Symposia on Molecular and Cellular Biology, Keystone, Colorado, April 2-8, 1998 (Keystone, Silverthorne, 1998).

3. Hugosson, M., Nurani, G., Glaser, E. \& Franzen, L.-G. Plant Mol. Biol. 28, 525-535 (1995).

4. Reinbothe, C., Ortel, B., Parthier, B. \& Reinbothe, S. Mol. Gen. Genet. 245, 616-622 (1994).
} 